\title{
Measuring renal function in acute heart failure: A place for old and new equations is
}

\author{
Margarida Alvelos ${ }^{\mathrm{a}, \mathrm{b}, *}$, Marta Couto ${ }^{\mathrm{a}, \mathrm{b}}$, Olga Laszczyńska ${ }^{\mathrm{c}}$, Pedro B. Almeida ${ }^{\mathrm{d}}$, João Guimarães ${ }^{\text {c,e,f }}$, \\ Ana Azevedo c,g, Paulo Bettencourt ${ }^{\mathrm{a}, \mathrm{b}}$
}

a Department of Internal Medicine, Centro Hospitalar de São João, Porto, Portugal

${ }^{\mathrm{b}}$ Department of Medicine, University of Porto Medical School, Porto, Portugal

c EPIUnit, Institute of Public Health of the University of Porto, Porto, Portugal

d Department of Cardiology, Centro Hospitalar de São João, Porto, Portugal

e Department of Clinical Pathology, Centro Hospitalar de São João, Porto, Portugal

${ }^{\mathrm{f}}$ Department of Biochemistry, University of Porto Medical School, Porto, Portugal

${ }^{g}$ Department of Clinical Epidemiology, Predictive Medicine and Public Health, University of Porto Medical School, Porto, Portugal

\section{A R T I C L E I N F O}

\section{Article history:}

Received 24 May 2015

Accepted 29 May 2015

Available online 31 May 2015

\section{Keywords:}

Heart failure

Glomerular filtration rate

Cystatin C

Prognosis

Renal dysfunction

Renal function impairment is frequent in acute heart failure (HF) episodes and is one of the most powerful determinants of outcome [1].

Glomerular filtration rate (GFR) is a widely accepted measure of renal function, and it may be estimated using several equations. The most frequently used are based on serum creatinine, although they are less accurate in over- and underweight patients, and in the extremes of renal function, since they tend to overestimate lower filtration rates and underestimate more elevated filtration rates [2]. Recent research has moved the focus towards potentially better and more sensitive markers of kidney function. Cystatin $C$ has arisen as an interesting alternative, and cystatin C-based equations estimating GFR have been developed, and, when compared to creatinine and creatinine-based equations, provide similarly or even more accurate measures of GFR [3].

is Grant support: This work was supported by two grants from "Fundação para a Ciência e a Tecnologia" [projects PIC/IC/82773/2007 and PTDC/SAU-ESA/107940/2008].

* Corresponding author at: Centro Hospitalar de São João, Alameda Professor Hernâni Monteiro, 4200-319 Porto, Portugal.

E-mail address: mpalvelos@gmail.com (M. Alvelos).
We aimed to assess the discrimination and predictive value of creatinine- and cystatin C-based estimated GFR (eGFR) equations, as well as a combined cystatin C- and creatinine-based eGFR equation, in predicting death in acute HF patients within six months after hospital discharge. Additionally we intended to determine the added value of cystatin $C$ in risk stratification in this population, compared to creatinine-based eGFR alone.

We prospectively enrolled patients consecutively admitted with acute HF, who were discharged alive, excluding those with acute coronary syndromes or on chronic renal function replacement therapy. Fasting venous blood samples were collected on discharge day. We used four creatinine-based GFR estimating equations - Cockcroft-Gault, Modification of Diet in Renal Disease (MDRD-6), IDMS-traceable simplified MDRD (MDRD-4) and CKD-EPI creatinine (CKD-EPICr) - , two cystatin C-based equations - from Rule et al. (CysCeq) [4] and CKD-EPI cystatin C (CKD-EPICysC) [3] - and an equation using both creatinine and cystatin C - CKD-EPI creatinine-cystatin C (CKD-EPICrCysC) [3]. The endpoint was 6-month all-cause death.

We used Kaplan-Meier to estimate the risk of 6-month all-cause death, according to the classes of eGFR for each equation, and Cox regression to quantify the association between eGFR categories and death. Net reclassification improvement (NRI) assessed the incremental value for predicting death when adding a cystatin C-based to a creatinine-based equation to classify GFR. Statistical analyses were performed using Stata 11.1 for Windows (StataCorp LP, College Station, TX).

The study protocol conforms to the ethical principles outlined in the Declaration of Helsinki, the local ethics committee approved the study, and informed consent was obtained.

Of the 478 patients included in the analysis $47 \%$ were men, the mean age was 75.6 years, and $41.9 \%$ had preserved ejection fraction. Mean serum creatinine and serum cystatin $\mathrm{C}$ at hospital discharge were $1.53 \mathrm{mg} / \mathrm{dL}$ and $1.64 \mathrm{mg} / \mathrm{L}$, respectively. Ninety patients (18.8\%) died during follow-up.

The distribution of patients across eGFR groups, according to the equations, is presented in Table 1. Using Spearman's correlation and Kappa coefficient, we found that the estimates from creatinine-based 
Table 1

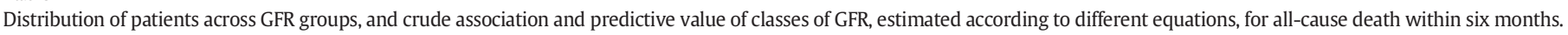

\begin{tabular}{|c|c|c|c|c|c|c|}
\hline & & \multicolumn{5}{|c|}{ eGFR $\left(\mathrm{mL} / \mathrm{min} / 1.73 \mathrm{~m}^{2}\right)$} \\
\hline & & $\geq 90$ & $60-89$ & $30-59$ & $<30$ & $\mathrm{p}$ for trend \\
\hline \multirow[t]{3}{*}{ Cockcroft-Gault } & $\mathrm{n}(\%)$ & $18(3.8)$ & $66(13.8)$ & $255(53.4)$ & $139(29.1)$ & $<0.001$ \\
\hline & $6 \mathrm{~m}(\%)$ & 6.7 & 6.2 & 17.5 & 29.8 & \\
\hline & $\mathrm{HR}(95 \% \mathrm{CI})$ & 1 & $1.13(0.13-10.09)$ & $3.41(0.47-24.74)$ & $6.24(0.86-45.39)$ & \\
\hline \multirow[t]{3}{*}{ MDRD-4 } & $\mathrm{n}(\%)$ & $16(3.4)$ & $85(17.8)$ & $281(58.8)$ & $96(20.1)$ & 0.057 \\
\hline & $6 \mathrm{~m}(\%)$ & 6.7 & 19.0 & 16.9 & 27.5 & \\
\hline & $\mathrm{HR}(95 \% \mathrm{CI})$ & 1 & $3.37(0.45-25.39)$ & $2.96(0.41-21.48)$ & $4.94(0.67-36.41)$ & \\
\hline \multirow[t]{3}{*}{ MDRD-6 } & $\mathrm{n}(\%)$ & $27(5.7)$ & $126(26.5)$ & $242(50.8)$ & $81(17.0)$ & 0.001 \\
\hline & $6 \mathrm{~m}(\%)$ & 11.6 & 12.8 & 18.9 & 32.4 & \\
\hline & $\mathrm{HR}(95 \% \mathrm{CI})$ & 1 & $1.21(0.35-4.15)$ & $1.83(0.57-5.89)$ & $3.36(1.02-11.11)$ & \\
\hline \multirow[t]{3}{*}{ CKD-EPICr } & $\mathrm{n}(\%)$ & $17(3.6)$ & $59(12.3)$ & $230(48.1)$ & $172(36.0)$ & 0.012 \\
\hline & $6 \mathrm{~m}(\%)$ & 6.3 & 15.3 & 16.3 & 25.3 & \\
\hline & $\mathrm{HR}(95 \% \mathrm{CI})$ & 1 & $2.82(0.36-22.28)$ & $3.03(0.42-22.09)$ & $4.84(0.67-35.14)$ & \\
\hline \multirow[t]{3}{*}{ CysCeq } & $\mathrm{n}(\%)$ & $21(4.4)$ & $92(19.3)$ & $231(48.3)$ & $134(28.0)$ & \\
\hline & $6 \mathrm{~m}(\%)$ & 0 & 10.2 & 16.3 & 33.0 & \\
\hline & $\mathrm{HR}(95 \% \mathrm{CI})$ & 1 & & $2.14(1.03-4.44)$ & $4.80(2.34-9.83)$ & $<0.001$ \\
\hline \multirow[t]{3}{*}{ CKD-EPICysC } & $\mathrm{n}(\%)$ & $39(8.2)$ & $88(18.4)$ & $220(46.0)$ & $131(27.4)$ & $<0.001$ \\
\hline & $6 \mathrm{~m}(\%)$ & 7.8 & 10.5 & 16.2 & 33.0 & \\
\hline & $\mathrm{HR}(95 \% \mathrm{CI})$ & 1 & $1.39(0.38-5.14)$ & $2.24(0.69-7.28)$ & $5.09(1.58-16.42)$ & \\
\hline \multirow[t]{3}{*}{ CKD-EPICrCysC } & $\mathrm{n}(\%)$ & $27(5.7)$ & $91(19.0)$ & $245(51.3)$ & $115(24.1)$ & $<0.001$ \\
\hline & $6 \mathrm{~m}(\%)$ & 4.0 & 13.4 & 17.8 & 29.8 & \\
\hline & $\mathrm{HR}(95 \% \mathrm{CI})$ & 1 & $3.85(0.50-29.62)$ & $5.32(0.73-38.62)$ & $9.47(1.30-69.18)$ & \\
\hline
\end{tabular}

eGFR $=$ estimated glomerular filtration rate.

Cockcroft-Gault $=$ Cockcroft-Gault equation.

MDRD-4 = simplified Modification of Diet in Renal Disease equation.

MDRD-6 = Modification of Diet in Renal Disease equation.

$\mathrm{CKD}-\mathrm{EPICr}=$ Chronic Kidney Disease Epidemiology Collaboration creatinine equation.

CysCeq = cystatin C-based equation from Rule et al.

CKD-EPICysC $=$ Chronic Kidney Disease Epidemiology Collaboration cystatin C equation

CKD-EPICrCysC = Chronic Kidney Disease Epidemiology Collaboration creatinine-cystatin C equation.

$6 \mathrm{~m}=6$-month risk of all-cause death.

$\mathrm{HR}=$ Crude Hazard Ratio.

$95 \% \mathrm{CI}=95 \%$ confidence interval.

equations were moderately correlated with each other and with estimates from cystatin C-based equations. The two equations using cystatin $\mathrm{C}$ were strongly correlated with each other and with the combined equation (data not shown).

The risk of death increased progressively across categories of eGFR for all equations (Table 1). Among the creatinine-based equations, Cockcroft-Gault provided the largest increase in risk of death. Cystatin C-based equations performed similarly, with a strong increase in risk across categories of eGFR. The combined equation yielded the largest increase in the predicted risk of death.

Given that cystatin $C$ is less readily available, we assessed the added value by subgroups of Cockcroft-Gault eGFR. Whereas for eGFR $<30$ or $\geq 60 \mathrm{~mL} / \mathrm{min} / 1.73 \mathrm{~m}^{2}$ according to Cockcroft-Gault, cystatin C or cystatin C-based eGFR did not discriminate prognosis further, patients who had Cockcroft-Gault eGFR 30-59 mL/min/1.73 $\mathrm{m}^{2}$ were reclassified by CKD-EPICysC equation into three subgroups of patients with different outcomes (Fig. 1). The incremental prognostic predictive ability of a cystatin C-based equation was further analysed by measuring the NRI, compared to Cockcroft-Gault alone. The addition of CKDEPICysC in this group of patients showed a significant improvement in risk prediction for 6-month all-cause mortality over CockcroftGault: NRI $=46 \%$ (95\% confidence interval: 23 to 69, $\mathrm{p}<0.001$ ). CysCeq yielded a NRI $=48 \%$ (95\% confidence interval: 25 to $71, \mathrm{p}<0.001$ ). The addition of the combined equation did not show a significant improvement in risk prediction ability.

The prognostic value of eGFR calculated by different equations in HF patients has been previously assessed, with findings of superior accuracy of CKD-EPICr over MDRD-4 [5]. These equations have also been compared with Cockcroft-Gault in chronic HF [6], and the latter revealed to be the best estimator of renal function for the prediction of long-term mortality, the same as in our work.

When compared to creatinine and creatinine-based eGFR equations, cystatin $\mathrm{C}$ is a similarly good marker of prognosis stratification in HF patients, particularly in those with moderate renal dysfunction [7]. A better prognostic value of cystatin C-based over creatinine-based equations has been observed in diverse populations. In a recent metaanalysis of 11 general population studies and 5 cohorts with chronic kidney disease, the use of eGFR based on cystatin C alone or in combination with creatinine, strengthened the association between the eGFR and the risks of death and end-stage renal disease [8]. In chronic HF, cystatin C-based also showed superior prognostic accuracy over creatinine-only based equations, particularly in patients with moderate renal dysfunction [9]. The utility of these equations in prognosis

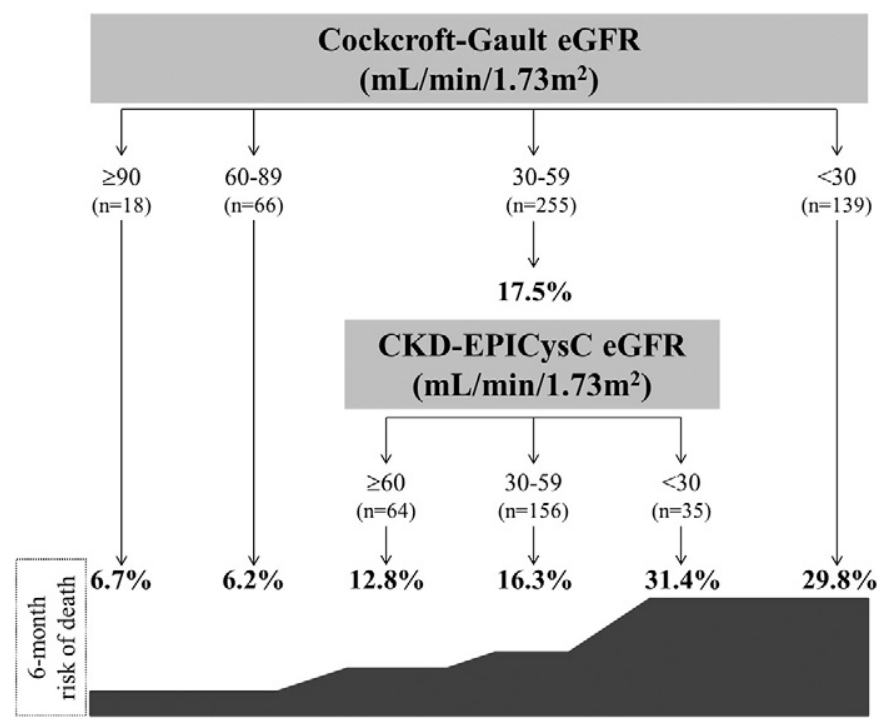

Fig. 1. Risk prediction for all-cause death within six months, based on the additive value of Cockcroft-Gault and CKD-EPICysC estimates of glomerular filtration rate. 
stratification of acute HF patients only recently has been addressed [10], and CKD-EPICysC and CKD-EPICrCysC were both superior to MDRD-4 for predicting long-term mortality and/or HF hospitalisation. However, contrarily to our findings, the better prognostic power of cystatin C-based equations was seen in patients with eGFR $\geq 60 \mathrm{~mL} / \mathrm{min} /$ $1.73 \mathrm{~m}^{2}$. These apparently conflicting results may be related to the fact that the creatinine-based equation used was MDRD-4, an equation that has better performance in patients with lower filtration rates.

Creatinine-based estimates of GFR will continue to be used in clinical practice, particularly given its low cost and universal availability. Nevertheless, we demonstrated that cystatin C-based estimation of GFR can refine the prognostic exercise of clinicians in acute HF patients with moderate renal dysfunction at hospital discharge.

\section{Conflicts of interest}

The authors report no relationships that could be construed as a conflict of interest.

\section{References}

[1] H.L. Hillege, D. Nitsch, M.A. Pfeffer, on behalf of the CHARM investigators, et al., Renal function as a predictor of outcome in a broad spectrum of patients with heart failure, Circulation 113 (2006) 671-678.
[2] T.D.J. Smilde, D.J. Veldhuisen, G. Navis, A.A. Voors, H.L. Hillege, Drawbacks and prognostic value of equations estimating renal function in patients with chronic heart failure and systolic dysfunction, Circulation 114 (15) (2006) 1572-1580.

[3] L.A. Inker, C.H. Schmid, H. Tighiouart, for the CKD-EPI Investigators, et al., Estimating glomerular filtration rate from serum creatinine and cystatin C, N. Engl. J. Med. 367 (2012) 20-29.

[4] A.D. Rule, E.J. Bergstralh, J.M. Slezak, J. Bergert, T.S. Larson, Glomerular filtration rate estimated by cystatin C among different clinical presentations, Kidney Int. 69 (2006) 399-405.

[5] M.A.E. Valente, H.L. Hillege, G. Navis, et al., The Chronic Kidney Disease Epidemiology Collaboration equation outperforms the Modification of Diet in Renal Disease equation for estimating glomerular filtration rate in chronic systolic heart failure, Eur. J. Heart Fail. 16 (1) (2014) 86-94.

[6] E. Zamora, J. Lupón, J. Vila, et al., Estimated glomerular filtration rate and prognosis in heart failure. Value of the Modification of Diet in Renal Disease Study-4, Chronic Kidney Disease Epidemiology Collaboration, and Cockcroft-Gault Equations, J. Am. Coll. Cardiol. 59 (19) (2012) 1709-1715.

[7] H. Naruse, J. Ishii, T. Kawai, et al., Cystatin C in acute heart failure without advanced renal impairment, Am. J. Med. 122 (2009) 566-573.

[8] M.G. Shlipak, K. Matsushita, J. Ärnlöv, for the CKD Prognosis Consortium, et al., Cystatin $C$ versus creatinine in determining risk based on kidney function, N. Engl. J. Med. 369 (2013) 932-943.

[9] E. Zamora, J. Lupton, M. de Antonio, et al., Long-term prognostic value for patients with chronic heart failure of estimated glomerular filtration rate calculated with the new CKD-EPI equations containing cystatin C, Clin. Chem. 60 (3) (2014) 481-489.

[10] S. Manzano-Fernández, P.J. Flores-Blanco, J.I. Pérez-Calvo, et al., Comparison of risk prediction with the CKD-EPI and MDRD equations in acute decompensated heart failure, J. Card. Fail. 19 (2013) 583-591. 